\title{
Targeting TR4 nuclear receptor suppresses prostate cancer invasion via reduction of infiltrating macrophages with alteration of the TIMP-1/MMP2/MMP9 signals
}

Xianfan Ding ${ }^{1,2+}$, Dong-Rong Yang ${ }^{2,3 \dagger}$, Liqun Xia ${ }^{1 \dagger}$, Bide Chen ${ }^{1}$, Shicheng Yu', Yuanjie Niư ${ }^{4}$, Mingchao Wang ${ }^{1}$, Gonghui Li ${ }^{*}$ and Chawnshang Chang ${ }^{2,5^{*}}$

\begin{abstract}
Background: TR4 nuclear receptor 4 (TR4) plays an important role in macrophages-associated foam cell formation of cardiovascular diseases and infiltrating macrophages are critical for prostate cancer (PCa) progression. However, the linkage of macrophages and TR4 and their impacts on PCa metastasis remains unclear.

Results: Knocking-down TR4 in human PCa cells (C4-2, CWR22Rv1), but not in human macrophages cells (THP-1), led to suppress the macrophages infiltration to PCa cells. The consequences of such suppression of the recruitment of macrophages toward PCa then resulted in suppressing the PCa cell invasion. Mechanism dissection found that knocking-down TR4 in PCa cells suppressed metastasis-related genes including MMP2, with induction of TIMP-1. Interruption assays using TIMP-1 neutralizing antibody could then reverse TR4-macrophage-mediated PCa invasion. IHC staining showed higher TR4 level, more macrophage infiltration, lower TIMP-1 and stronger MMP2/MMP9 in tumor tissues of the Gleason score $5+4$ patients compared with the Gleason score $3+3$ patients.
\end{abstract}

Conclusion: Targeting TR4 in prostate tumor microenvironment might represent a potential new therapeutic approach to better battle PCa metastasis.

Keywords: TR4, Macrophage, Prostate cancer, Invasion, TIMP-1

\section{Background}

Prostate cancer $(\mathrm{PCa})$ progression may be influenced by many cells co-existing in the tumor microenvironment that includes several types of immune cells [1-4]. These infiltrating immune cells may secrete various cytokines or chemokines to affect the PCa progression. In return, $\mathrm{PCa}$ cells may also be able to secrete different cytokines or chemokines to influence the migration of these infiltrating immune cells toward PCa. The consequences of these mutual interactions between $\mathrm{PCa}$ cells and infiltrating

\footnotetext{
* Correspondence: ligonghui1970@hotmail.com; chang@urmc.rochester.edu ${ }^{\dagger}$ Equal contributors

${ }^{1}$ Department of Urology and Chawnshang Chang Liver Cancer Center, Sir Run Run Shaw Hospital, School of Medicine, Zhejiang University, Hangzhou 310016, China

${ }^{2}$ George Whipple Lab for Cancer Research, Departments of Pathology, Urology and Radiation Oncology, and The Wilmot Cancer Center, University of Rochester Medical Center, Rochester, NY 14646, USA

Full list of author information is available at the end of the article
}

immune cells may then alter the PCa cell proliferation, invasion and survival [5-8]. Among many infiltrating immune cells, macrophages have recently been demonstrated to play important roles to promote $\mathrm{PCa}$ initiation [1] and invasion $[2,3]$.

Testicular nuclear receptor 4 (TR4) is a member of the nuclear receptor superfamily that functions as a transcriptional regulator to modulate its downstream target genes for its influence on selective diseases [9-13] including PCa [14], metabolic syndrome and cardiovascular diseases [15-18], as well as aging [19], cerebellar development [9], and fertility [20,21]. Importantly, TR4 could also play a critical role in macrophages-associated foam cell formation of cardiovascular diseases [22] and mycobacterium tuberculosis disease [23]. However, the linkage of macrophages and TR4 and their influence on PCa metastasis remains unclear. 
Here we applied the co-culture invasion system with human macrophage THP-1 cells and human castration resistant prostate cancer (CRPC) C4-2 and CWR22Rv-1 cells to demonstrate that targeting TR4 via TR4-siRNA suppresses the recruitment of infiltrating macrophages and the consequences of such suppression may then lead to inhibit the Pca invasion.

\section{Results}

Knocking-down TR4 alters the macrophage migration in PCa-macrophages co-culture system

We first applied the in vitro macrophage recruitment assays with co-culture of human PCa C4-2 cells and human THP-1 monocytes/macrophages to study the effect on macrophage recruitment to PCa cells. We collected

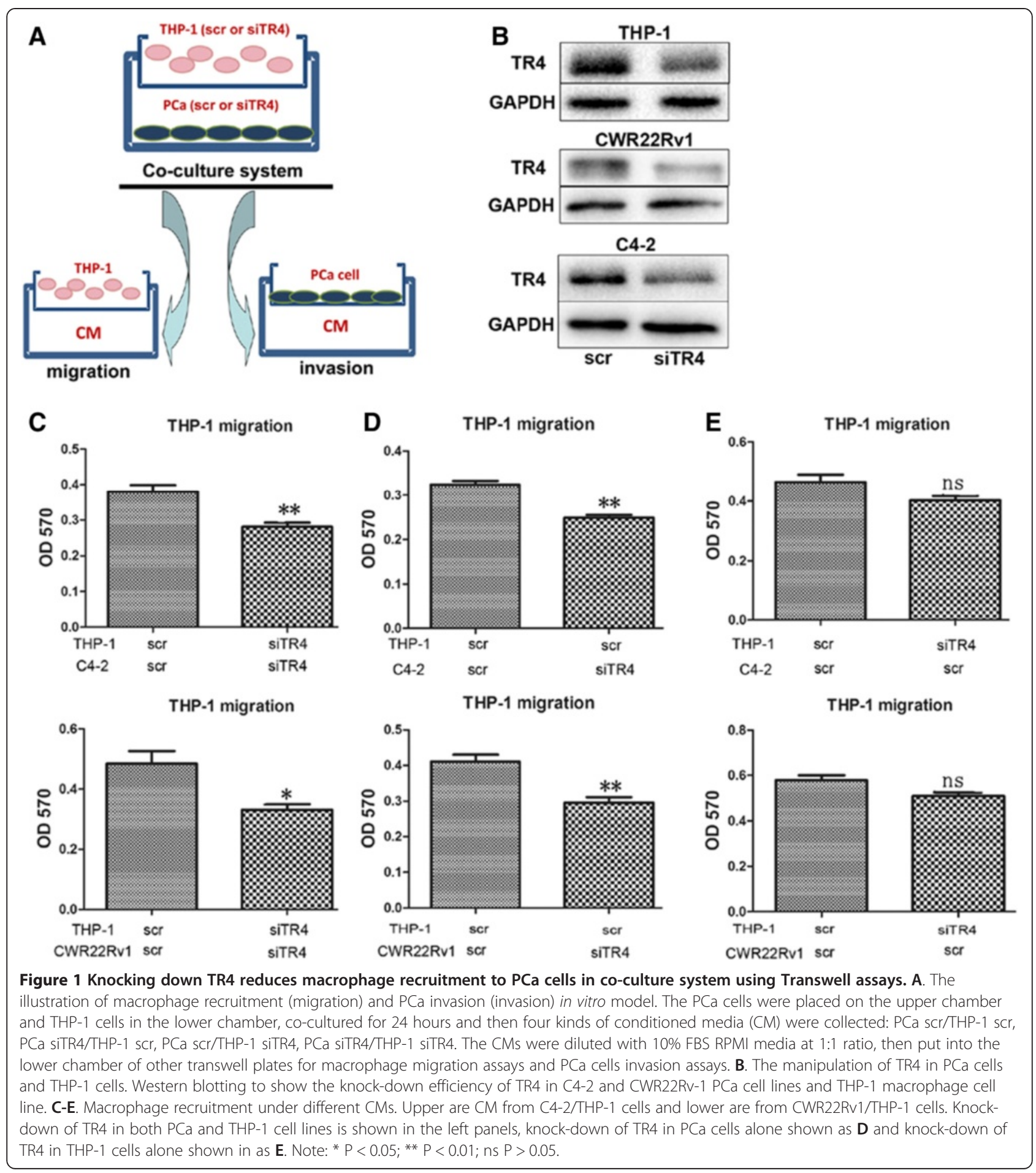


three different conditioned media(CM) from C4-2 cells cultured alone, THP-1 cells cultured alone and C4-2 and THP-1 co-cultured, and results showed the CM from co-cultured C4-2 and THP-1 cells had stronger influence on recruiting THP-1 cells (Additional file 1: Figure S1).

The PCa scr (scramble)/siTR4 cells were co-cultured with THP-1 scr/siTR4 cells (Figure 1A, upper) respectively for 24 hours in the $0.4 \mu \mathrm{M}$ transwell plates, and CMs were collected and diluted with regular media before applying in the human THP-1 macrophage recruitment assay in the $5 \mu \mathrm{M}$ transwell plate system (Figure 1A, lower and left). The manipulation of TR4 protein levels were shown in Figure 1B.
The results revealed that the CM from the PCa siTR4/ THP-1 siTR4 co-culture significantly decreased the macrophage recruitment (Figure 1C). Importantly, we found knocked-down TR4 in PCa cells also led to similar results showing decreased macrophage recruitment to the CM of PCa siTR4/THP-1 scr co-culture system (Figure 1D). In contrast, we observed a less obvious effect when we knocked-down TR4 in THP-1 cells only (Figure 1E).

Together, results from Figure 1A-E and Additional file 1: Figure S1 suggest that knocking-down TR4 in PCa cells, and not in macrophage THP-1 cells, led to suppress macrophage recruitment to the $\mathrm{CM}$ from $\mathrm{PCa}$ siTR4/THP-1 scr (or THP-1 siTR4) co-culture system.
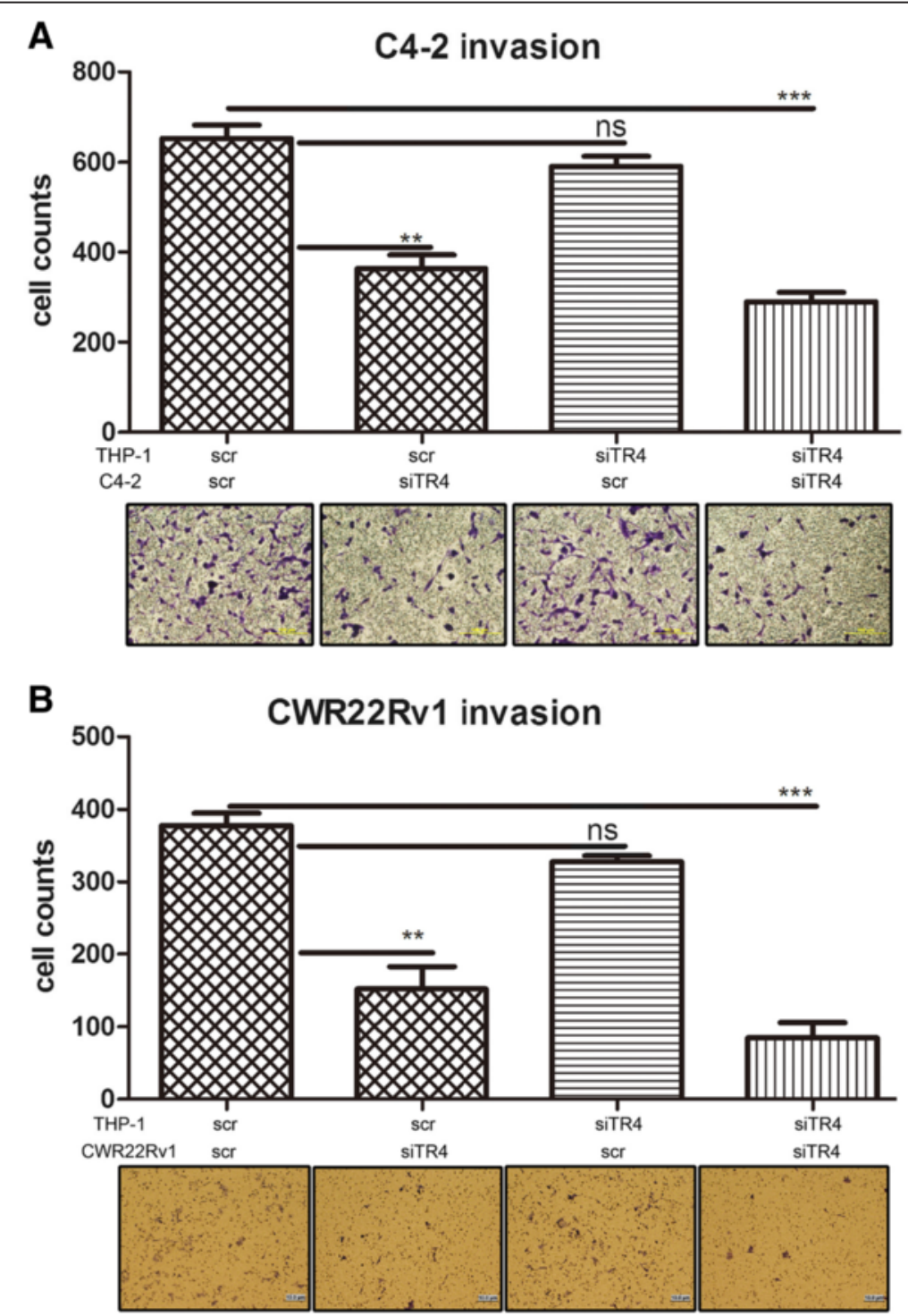

Figure 2 PCa cells invasion assay under different CMs conditions. A. C4-2 cells invasion assay; B. CWR22Rv1 cells invasion assay. Parental PCa cell invasion ability using different CMs from: PCa scr/THP-1 scr, PCa siTR4/THP-1 scr, PCa scr/THP-1 siTR4, PCa siTR4/THP-1 siTR4 (left to right). Lower pictures are images and upper columns are cell counts. 
Reduced macrophage migration suppressed PCa invasion To determine the consequences after suppression the recruited macrophages, we applied co-culture system with $8 \mu \mathrm{M}$ transwell plates to measure the invasion ability of PCa parental cells under different CMs. The CMs were prepared as in Figure 1 and put into the lower chambers of the transwell plates. The parental C4-2 or CWR22Rv1 cells were placed on the upper chambers with membranes pre-coated with matrigel for the $\mathrm{PCa}$ invasion assay (Figure $1 \mathrm{~A}$, lower and right). As shown in Figure $2 \mathrm{~A}-\mathrm{B}$, we found the $\mathrm{CM}$ from the $\mathrm{PCa}$ siTR4/THP-1 siTR4 co-cultured cells significantly decreased the PCa invasion in both C4-2 (Figure 2A) and CWR22Rv1 (Figure 2B) cells tested. Similarly, CM from knocking-down TR4 in PCa cells only could also suppress PCa cells invasion. In contrast, we found no significant effect when we knocked-down TR4 in only THP-1 cells (Figure 2).

Together, results from Figures 1 and 2 demonstrated that knocked-down TR4 in PCa cells might reduce the macrophage infiltration to PCa cells to suppress PCacells invasion.

TIMP-1/MMP2/MMP9 signals was altered significantly in the parental PCa cells treated with the CM from PCa siTR4/THP-1 siTR4 co-cultured system
To dissect the potential molecular mechanism why targeting TR4 could suppress PCa invasion via suppression of macrophage recruitment, we screened tumor metastasis-related genes using qPCR gene microarray. We found that the TIMP-1 mRNA expression increased in the parental PCa cells treated with the CM from PCa siTR4/THP-1 siTR4 co-culture system when compared with the CM from PCa scr/THP-1 scr co-culture system. Interestingly, the expression of other two metastasisrelated genes (MMP2 and MMP9) was decreased (Figure 3A). Western blotting also confirmed the altered protein expression of TIMP-1/MMP2/MMP9. 9 (Figure 3B). As TIMP-1 could function as suppressor for MMP2 and MMP9 [24,25], TIMP-1 to MMP2/ MMP9 signals may be the key factor to reduce the parental PCa cells invasion treated with the CM from PCa siTR4/THP-1 siTR4 co-culture system.

\section{TIMP-1 antibody reversed the knocked-down TR4 effect} on PCa invasion

To confirm the results above, we then applied an interruption assay to see if blocking TIMP-1 to MMP2/ MMP9 signals with neutralizing anti-TIMP-1 antibody might alter the TR4 ability to influence PCa invasion. As shown in Figure 4, addition of neutralizing anti-TIMP-1

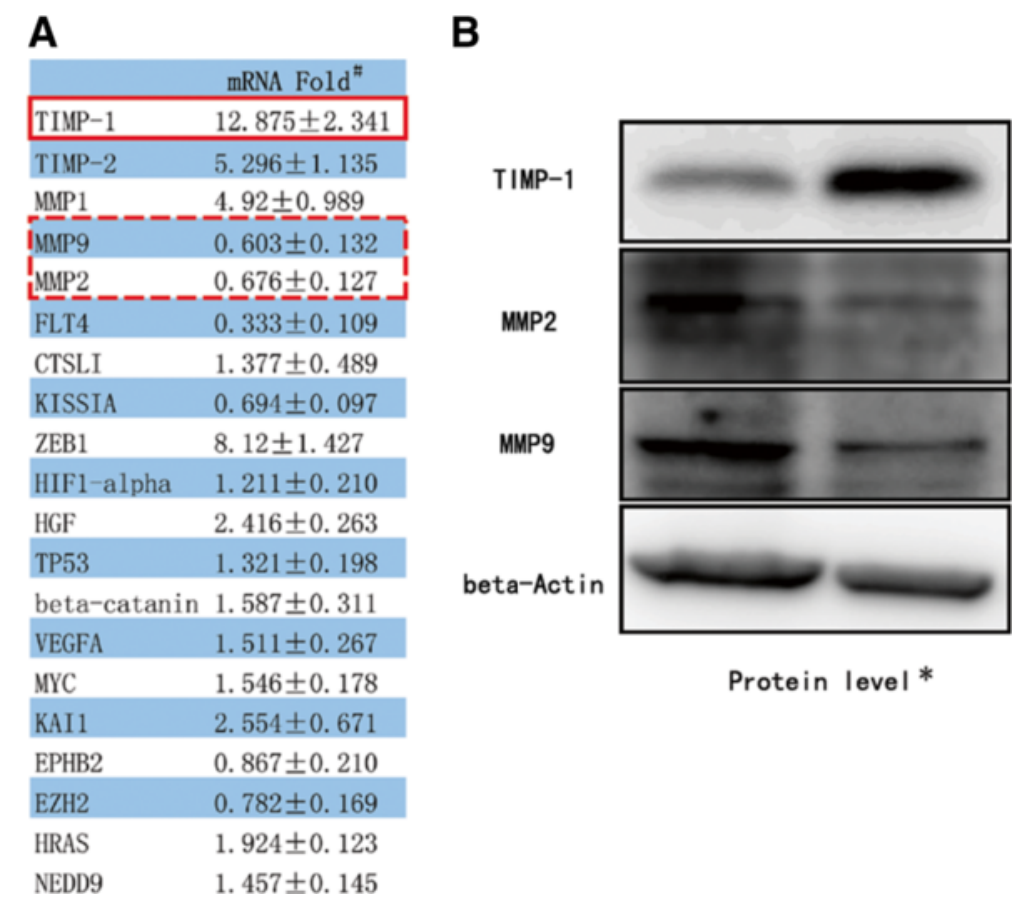

Figure 3 TIMP-1/MMP2,9 singaling showed obvious change when parental C4-2 cells cultured in the CM from THP-1 siTR/C4-2 siTR4. A: mRNA level of qPC Rarray; B: Protein level of TIMP-1/MMP2/MMP9 signaling. * Parental C4-2 cells were treated for 24 hours with the CMs from the co-culture of PCascr/THP-1 scr (control) and C4-2 siTR4/THP-1 siTR4 (study). mRNA was isolated and then metastasis-related genes qPCR array were applied to screen for the potential factors in knocked-down of TR4-reduced macrophage recruitment-PCa cells invasion. These folds represent the ratio of study/control. \# Western blotting to confirm the change of TIMP-1/MMP2/MMP9 signaling. Left line is control group, right line is study group. 

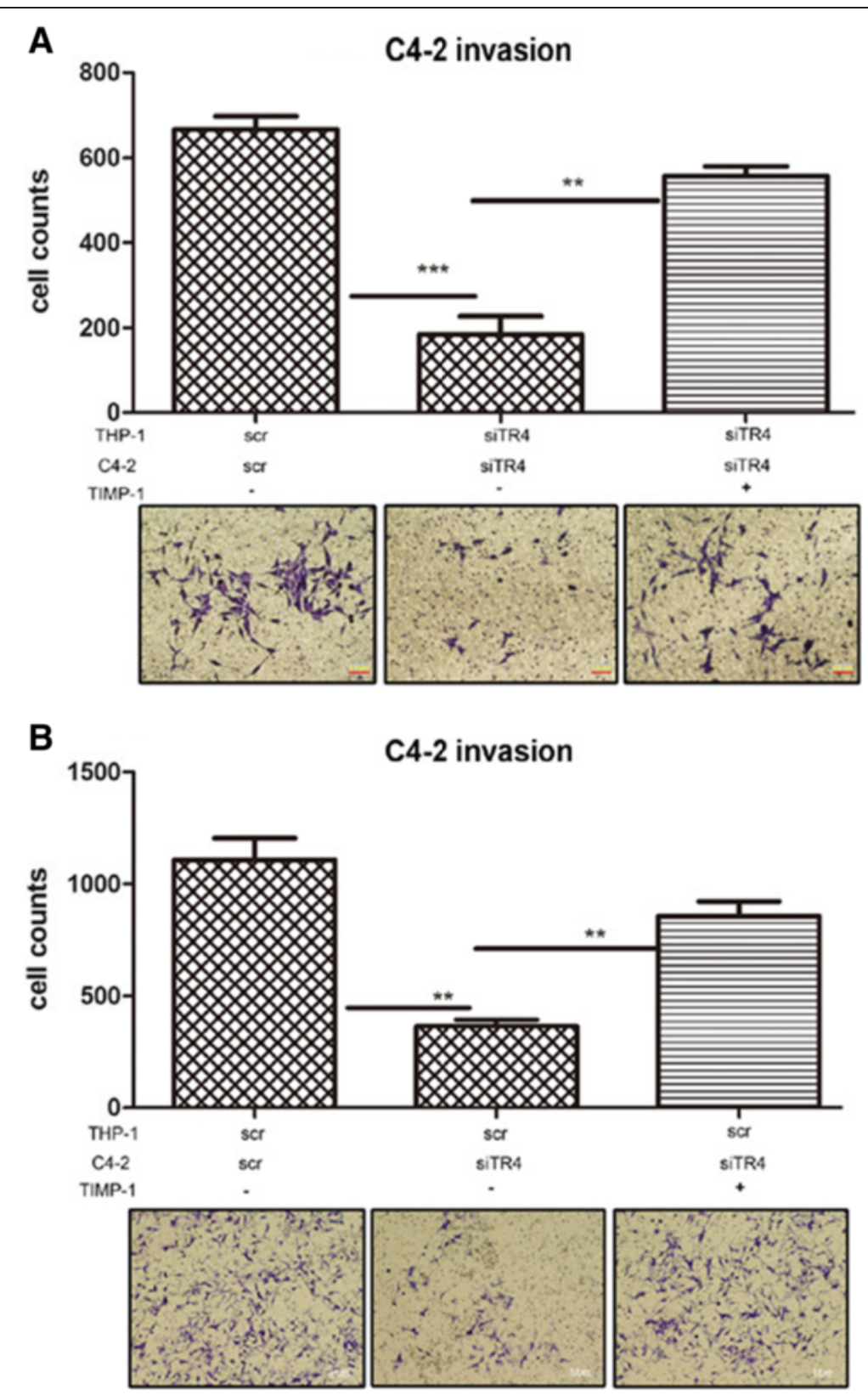

Figure 4 Neutralization study with TIMP-1 antibody. TIMP-1 neutralization antibody or IgG, as vehicle control, were added into the CMs in lower chamber to study PCa cells invasion ability. A. CM: PCa siTR4/THP-1 siTR4; B. CM: PCa siTR4/THP-1 scr.

antibody reversed the TR4 ability to alter the C4-2 invasion. (Figure 4A: CM from C4-2 siTR4/THP-1 siTR4 coculture system; Figure 4B: CM from C4-2 siTR4/THP-1 scr co-culture system). Similar results were also found when we replaced C4-2 with CWR22RV-1 (Additional file 1: Figure S2).

Together, results from Figure 3, Figure 4 and Additional file 1: Figure S2 demonstrated that TIMP-1 to MMP2/9 signals might play key roles to mediate TR4-macrophages effects on PCa cell invasion.
Higher TR4 expression with more macrophages, lower TIMP-1, and stronger MMP2/MMP9 expression in human $\mathrm{PCa}$ of higher Gleason score

To further confirm our in vitro results above, we then examined the expression of TR4 and macrophage infiltration (marker: CD68) in clinical tissue samples obtained from PCa patients with different Gleason scores. We found higher TR4 expression in PCa with the Gleason score $5+4$ patients compared with those with Gleason score $3+3$ patients (Figure 5, first line), and more infiltrated 


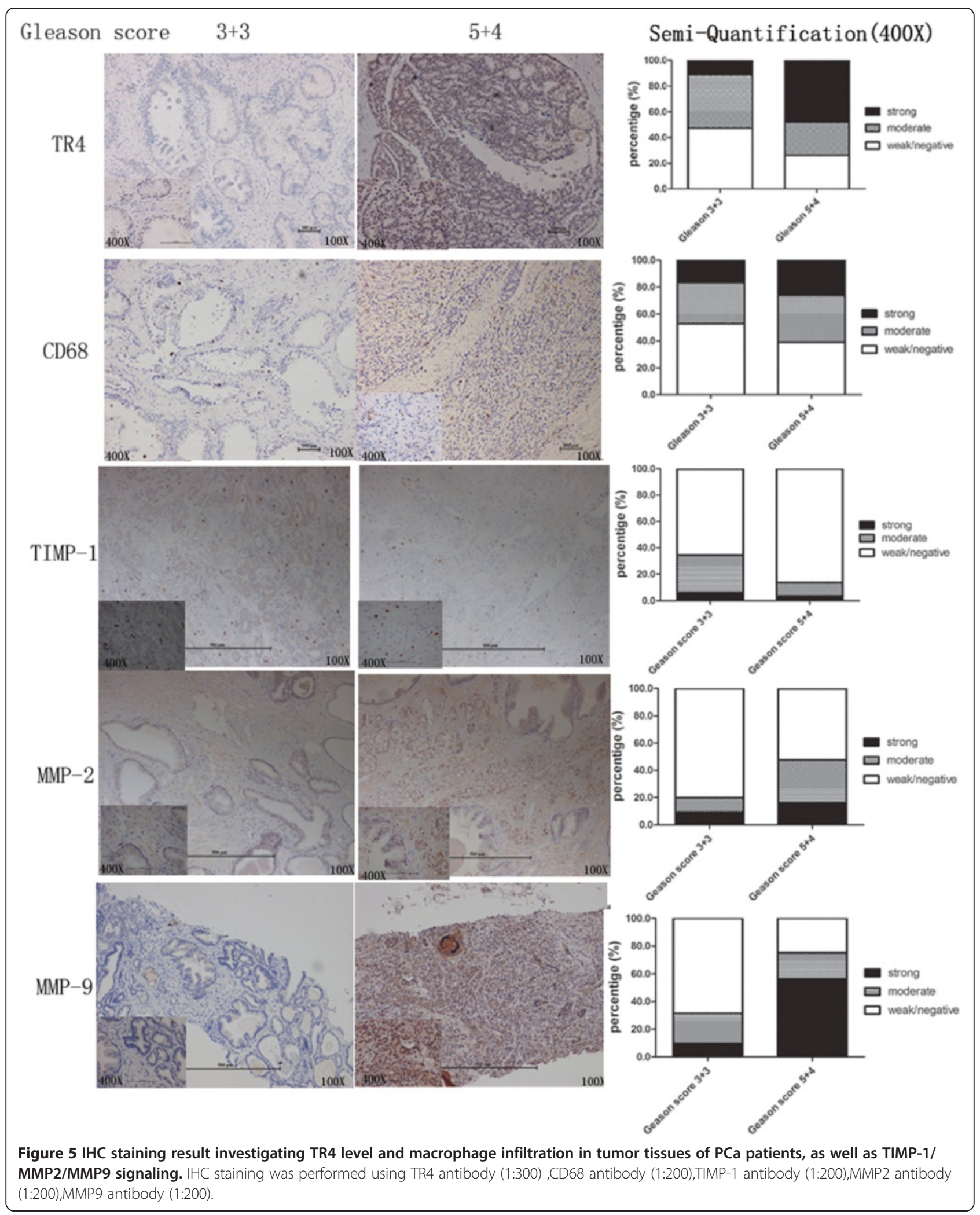


macrophages in PCa with the Gleason score $5+4$ patients (Figure 5, second line). Importantly, we also found lower TIMP-1 expression with higher MMP2/ MMP9 expression in PCa of Gleason score $5+4$, compared with Gleason score $3+3$ (Figure 5, third-fifth line).

Together, results from Figures 1, 2, 3, 4 and 5 demonstrate that TR4 expression is correlated with macrophage infiltration and PCa invasion that may involve the modulation the TIMP-1-MMP2/MMP9 signaling.

\section{Discussion}

Inflammation is a protective reaction of living tissue to internal or external environmental stimuli that aims to remove the invasive agents and restore the tissue physiology. Approximately, a quarter of tumors may attribute to chronic infections and other types of unresolved inflammation [5]. The inflammation present in the tumor microenvironment is characterized by leukocyte infiltration, ranging in size, distribution and composition, and may include the tumor-associated macrophages mast cells, dendritic cells, natural killer cells, neutrophils and lymphocytes [5-8]. Macrophages are among the first immune cells to infiltrate the tumor lesions and then influence the tumor progression/invasion via secretion of several growth factors, cytokines or chemokines, including colony stimulating factor 1 (CSF-1), vascular epithelial growth factor (VEGF) and CCL2 [3,5-8]. Recent studies also demonstrated that targeting the androgen receptor with siRNA might lead to enhance PCa invasion via increasing the recruitment of infiltrating macrophages that involved the modulation of CCL2-STAT3 signaling [3].

The imbalance between MMPs and their tissue inhibitors TIMPs have been implicated significantly in PCa progression, and usually the observed imbalance of MMPsTIMPs could be due to a loss of TIMP-1 [26]. TIMP-1 may function as suppressor to inhibit several metastasis genes, such as MMP2 and MMP9. Many studies also documented well that TIMP-1 and MMP2/MMP9 could be related to the invasive/metastatic ability of PCa [26-28].

Xie et al. [22] reported that foam cell formation was reduced in TR4 knockout (TR4 ${ }^{-/-}$) mice via manipulation of CD36 expression in cardiovascular diseases. Mahajan et al. [23] also found M. tuberculosis macrophage lipids could interact with TR4 to ensure survival of the pathogen by modulating macrophage function. Our current study also found an important role of TR4 in tumor associated macrophages: TR4 in PCa cells may play a positive role to attract macrophage infiltration, the consequent crosstalk between PCa cells and macrophage may produce some changes in the tumor microenvironment around PCa cells, and these changes may alter the invading ability of PCa cells via different mechanisms. We were more interested with the molecular mechanism of decreased PCa cell invasion under the CM from the co-culture system of PCa siTR4/THP-1 siTR4. The further dissection found TIMP-1 level was increased when parental $\mathrm{PCa}$ cells were cultured in the $\mathrm{CM}$ from C4-2 siTR4/THP-1 siTR4, and addition of neutralizing antibody of TIMP-1 reversed this effect. Together, these results suggest that TIMP-1-MMP2/9 signals may play key roles in the TR4-mediated macrophages infiltrationPCa cell invasion.

Other key genes related to the PCa invasion including TIMP-2, MMP1, and ZEB1 may be also involved in this macrophages-PCa co-culture system, and more detailed studies may be needed to further dissect their roles in mediating this infiltrated macrophages-PCa cell invasion.

In summary, these results suggest that targeting TR4 might suppress macrophage infiltration and consequently reduce the PCa cell invasion. Further studies via development of small molecules to target TR4 may help us to develop a potential new therapeutic approach to suppress this newly identified signal to better suppress the PCa at later metastatic stages.

\section{Methods}

\section{Cell culture}

Two human PCa cell lines C4-2, CWR22RV-1, and the human THP-1 cell line derived from leukemia cells were purchased from the American Type Culture Collection. PCa cells were maintained in RPMI 1640 (CWR22Rv-1) or DMEM (C4-2) containing 10\% FBS, THP-1 cells were maintained in RPMI1640 medium containing 10\% heatinactivated FBS, and $5 \mathrm{mM}$ 2-mercaptoethanol. All media contained $2 \mathrm{mM}$ L-glutamine, $100 \mathrm{IU} / \mathrm{ml}$ penicillin and $100 \mu \mathrm{g} / \mathrm{ml}$ streptomycin and all cells were incubated at $37^{\circ} \mathrm{C}$ in a humidified incubator at $5 \% \mathrm{CO} 2$.

\section{Antibodies}

Anti-TR4 (PP-0107B-00) and anti-TIMP-1 (AF970) antibodies were purchased from R\&D systems (Minneapolis, MN). Anti-GAPDH (6c5) antibody was purchased from Santa Cruz Biotechnology (Santa Cruz, CA). CD68 antibody (ab125212) was purchased from Abcam (Cambridge, MA).

\section{Reverse transcription (RT) and qPCR analysis}

Total RNAs were isolated from C4-2, and CWR22Rv-1 cells using TRIzol $^{\circ}$ Reagent (Invitrogen). RNA $(2 \mu \mathrm{g})$ was used as a template for the RT reaction $(20 \mu \mathrm{l}$ system). The RT reaction mixture (Invitrogen) contained $1 \mu \mathrm{l}(10$ $\mathrm{pM}$ ) of primers. The resultant cDNA was then used to carry out real time quantitative RT-PCR, using SYBR Green PCR MasterMix, on the iCycleriQ $^{\text {ma }}$ PCR cycler and detection system (Bio-Rad Laboratories, Hercules, CA). PCR conditions were as follows: $95^{\circ} \mathrm{C}$ for $5 \mathrm{~min}$, followed by 40 cycles at $95^{\circ} \mathrm{C}$ for $1 \mathrm{~min}, 55^{\circ} \mathrm{C}$ for $1 \mathrm{~min}$ 
and $72^{\circ} \mathrm{C}$ for $1 \mathrm{~min}$. The final extension was at $72^{\circ} \mathrm{C}$ for $5 \mathrm{~min}$. Calculation of relative gene expression was performed using the $2^{-\Delta \Delta} \mathrm{CT}$ method.

\section{THP-1 recruitment assay}

The $\mathrm{PCa} /$ macrophage cells were co-cultured in $0.4 \mu \mathrm{M}$ pore size transwell plates for 24 hours. Then the conditioned media $(\mathrm{CM})$ were collected, diluted with normal culture media, plated into the lower chamber of new 24well transwells with $5 \mu \mathrm{M}$ pore polycarbonate membrane insert (Corning, \#3422, BD Biosciences, San Jose, CA). $1 \times 10^{5}$ of parental THP- 1 cells were plated onto the upper chamber for macrophage migration assay. The cells migrated into the lower chamber were measured using 3(4,5-Dimethylthiazol-2-yl)-2,5-diphenyltetrazolium bromide (MTT)method. MTT solution $(100 \mathrm{~mL}, 5 \mathrm{mg} / \mathrm{mL}$ in PBS) was added to each well of the transwell plates and incubated for 2 hours at $37^{\circ} \mathrm{C}$. Centrifuged at $3000 \mathrm{rmp}$ for 20 minutes, discarded the supernatant discarded and $300 \mu \mathrm{l} /$ well dimethylsulfoxide (DMSO) was added to dissolve the formazan. The absorbance was immediately measured at $570 \mathrm{~nm}$ using a Bio-Rad Absorbance Reader. Each sample was assayed in triplicate and the experiments were repeated at least three times.

\section{Cell invasion assay}

$\mathrm{CM}$ was collected and diluted as above and added into the lower chamber of the 24-well transwell with $8 \mu \mathrm{M}$ pore polycarbonate membrane insert (Corning, \#3422) pre-coated with matrigel for cell invasion assay. The parental PCa cells were plated onto upper chamber at $1 \times 10^{5}$. Each sample was assayed in triplicate. The cells invaded to the lower chamber were fixed, stained using $1 \%$ toluidine blue, and the numbers averaged after counting 6 randomly selected fields. Each experiment was repeated at least in triplicate.

\section{Western blotting}

The cells were washed with $1 \mathrm{xPBS}$ and scraped into a lysis buffer containing the proteinase inhibitor cocktail (Roche). Protein concentrations were measured with the BCA protein reagent (Pierce Chemical, Rockford, IL). Approximately $50 \mu \mathrm{g}$ of protein/lane were loaded and run on the polyacrylamide gel with a Tris/glycine running buffer system and then transferred onto a polyvinylidene difluoride membrane. The primary antibodies with dilutions of 1:500 to 1:1,000 were added and then incubated overnight in the cold room with vigorous shaking. The horseradish peroxidase-conjugated secondary antibody (Pierce Chemical, Rockford, IL) was added and the signals were detected by adding the enhanced chemiluminescence Western blotting detection reagents (Amersham Biosciences, Piscataway, NJ).
Clinical samples and immunohistochemical (IHC) staining Clinical tissues of twenty-three PCa patients of Gleason score $5+4$ and thirty-six Gleason score $3+3 \mathrm{PCa}$ patients were collected from Sir Run Run Shaw Hospital. Tissues were fixed in $10 \%(\mathrm{v} / \mathrm{v})$ formaldehyde in PBS, embedded in paraffin, and cut into $4 \mu \mathrm{m}$ sections and used for IHC staining with human TR4/CD68 antibodies. To enhance antigen exposure, the slides were treated with $1 \times$ EDTA at $98^{\circ} \mathrm{C}$ for $10 \mathrm{~min}$ for antigen retrieval. The slides were incubated with endogenous peroxidase blocking solution to inhibit endogenous peroxidase, and then were incubated with the primary antibody at room temperature for $60 \mathrm{~min}$. After rinsing with Tris-buffered saline, the slides were incubated for $45 \mathrm{~min}$ with biotin-conjugated secondary antibody, washed, and then incubated with enzyme conjugate horseradish peroxidase (HRP)-streptavidin. Freshly prepared DAB (Zymed, South San Francisco, CA) was used as substrate to detect HRP. Finally, slides were counterstained with hematoxylin and mounted with aqueous mounting media. All the tissues were stained and examined by 2 independent pathologists in the same hospital in a blinded manner without prior knowledge of the clinical information. German Immunoreactive Score (IRS)was calculated to measure the protein levels. Briefly, the IRS assigns sub-scores for the percentage of immunoreactive cells (0-4) and immunoreactive intensity $(0-3)$, then multiplies them to yield the IRS score, which ranged from 0 to 12 . The percent positivity was scored as "0" (<1\%), "1" (1-10\%), "2" (11-50\%), "3" (51$80 \%)$, "4" (>80\%). The staining intensity was scored as "0" (negative), "1" (weak), "2" (moderate), and "3" (strong). Scores were considered negative (0-1), weakly positive (2-4), moderately positive (6-8), and strongly positive (9-12).

\section{Statistics}

Data are presented as the means \pm SD for the indicated number of separate experiments. The statistical significance of differences between two groups of data was analyzed by paired $t$-test and P-values $<0.05$ were considered significant.

\section{Additional file}

Additional file 1: Figure S1. The effect of CMs on macrophage recruitment. The $C 4-2\left(5 \times 10^{5}\right) / T H P-1\left(5 \times 10^{5}\right)$ cells were co-cultured in $0.4 \mu \mathrm{M}$ pore size transwell plates. $1 \times 10^{6} \mathrm{C} 4-2$ only or THP-1 only were inoculated into a 6-well plate. CMs of these three groups were collected after 24 hours culture. The CMs were diluted with freshmedia at 1:1 ratio then plated into the lower chamber of 24-well transwells with $5 \mu \mathrm{M}$ pore size. $1 \times 10^{5}$ parental THP-1 cells were plated onto the upper chamber for macrophage migration assay. Figure S2. Neutralization study with CWR22Rv-1. TIMP-1 neutralization antibody or lgG, as vehicle control, were added into the CMs in lower chamber to observe CW22Rv-1 cells invasion ability. 


\section{Competing interests}

The authors declare that they have no competing interests.

\section{Authors' contributions}

XD: Experiments, Writing the article; D-RY Experiments; L-QX: Writing the article; YN: Obtained funding; BC: Experiments; SY: Statistical analysis; MW: Writing the article; GL: Obtained funding, Conception and design; CC: Obtained funding, Overall responsibility. All authors read and approved the final manuscript.

\section{Acknowledgements}

We thank Karen Wolf for helping in the preparation of this manuscript. These studies were supported by National Basic Research 973 Program of China (2012CB518304); NIH (CA122840, CA156700); Zhejiang Provincial Natural Science Foundation of China (LQ14H050001); Medical Research Project of Hangzhou Health Bureau, China (2014A36); George Whipple Professorship Endowment; Taiwan Ministry of Health and Welfare Clinical Trial and Research Center of Excellence (DOH102-TD-B-111-004).

\section{Author details}

${ }^{1}$ Department of Urology and Chawnshang Chang Liver Cancer Center, Sir Run Run Shaw Hospital, School of Medicine, Zhejiang University, Hangzhou 310016, China. ${ }^{2}$ George Whipple Lab for Cancer Research, Departments of Pathology, Urology and Radiation Oncology, and The Wilmot Cancer Center, University of Rochester Medical Center, Rochester, NY 14646, USA. ${ }^{3}$ Department of Urology, the 2nd Affiliated Hospital of Soochow University, Suzhou 215004, China. ${ }^{4}$ Chawnshang Chang Sex Hormone Research Center, Tianjin Institute of Urology, Tianjin Medical University, Tianjin 300211, China. ${ }^{5}$ Sex Hormone Research Center, China Medical University/Hospital, Taichung 404, Taiwan.

Received: 16 April 2014 Accepted: 22 December 2014

Published online: 27 January 2015

\section{References}

1. Fang LY, Izumi K, Lai KP, Liang L, Li L, Miyamoto $H$, et al. Infiltrating macrophages promote prostate tumorigenesis via modulating androgen receptor-mediated CCL4-STAT3 signaling. Cancer Res. 2013;73:5633-46.

2. Izumi K, Fang LY, Mizokami A, Namiki M, Li L, Lin WJ, et al. Targeting the androgen receptor with siRNA promotes prostate cancer metastasis through enhanced macrophage recruitment via CCL2/CCR2-induced STAT3 activation. EMBO Mol Med. 2013;5:1383-401.

3. Lin TH, Lee SO, Niu Y, Xu D, Liang L, Li L, et al. Differential androgen deprivation therapies with anti-androgens casodex/bicalutamide or MDV3100/Enzalutamide versus anti-androgen receptor ASC-J9(R) Lead to promotion versus suppression of prostate cancer metastasis. J Biol Chem. 2013;288:19359-69.

4. Wang X, Lee SO, Xia S, Jiang Q, Luo J, Li L, et al. Endothelial cells enhance prostate cancer metastasis via IL-6-> androgen receptor-> TGF-beta- > MMP-9 signals. Mol Cancer Ther. 2013;12:1026-37.

5. Coussens LM, Werb Z. Inflammation and cancer. Nature. 2002;420:860-7.

6. De Marzo AM, Platz EA, Sutcliffe S, Xu J, Gronberg H, Drake CG, et al. Inflammation in prostate carcinogenesis. Nat Rev Cancer. 2007;7:256-69.

7. De Marzo AM, Nakai Y, Nelson WG. Inflammation, atrophy, and prostate carcinogenesis. Urol Oncol. 2007;25:398-400.

8. Zhu P, Baek SH, Bourk EM, Ohgi KA, Garcia-Bassets I, Sanjo H, et al. Macrophage/cancer cell interactions mediate hormone resistance by a nuclear receptor derepression pathway. Cell. 2006;124:615-29.

9. Ding XF, Yu SC, Chen BD, Lin SJ, Chang C, Li GH. Recent advances in the study of testicular nuclear receptor 4. J Zhejiang Univ Sci B. 2013;14:171-7.

10. Chang C, Da Silva SL, Ideta R, Lee Y, Yeh S, Burbach JP. Human and rat TR4 orphan receptors specify a subclass of the steroid receptor superfamily. Proc Natl Acad Sci U S A. 1994;91:6040-4.

11. Du L, Bergsneider M, Mirsadraei L, Young SH, Jonker JW, Downes M, et al. Evidence for orphan nuclear receptor TR4 in the etiology of Cushing disease. Proc Natl Acad Sci U S A. 2013;110:8555-60.

12. Lee YF, Young WJ, Lin WJ, Shyr CR, Chang C. Differential regulation of direct repeat 3 vitamin D3 and direct repeat 4 thyroid hormone signaling pathways by the human TR4 orphan receptor. J Biol Chem. 1999;274:16198-205.
13. Inui S, Lee YF, Haake AR, Goldsmith LA, Chang C. Induction of TR4 orphan receptor by retinoic acid in human $\mathrm{HaCaT}$ keratinocytes. J Invest Dermatol. 1999;112:426-31.

14. Yang DR, Ding XF, Luo J, Shan YX, Wang R, Lin SJ, et al. Increased chemosensitivity via targeting testicular nuclear receptor 4 (TR4)-Oct4interleukin 1 receptor antagonist (IL1Ra) axis in prostate cancer CD133+ stem/ progenitor cells to battle prostate cancer. J Biol Chem. 2013;288:16476-83.

15. Kang HS, Okamoto K, Kim YS, Takeda Y, Bortner CD, Dang H, et al. Nuclear orphan receptor TAK1/TR4-deficient mice are protected against obesitylinked inflammation, hepatic steatosis, and insulin resistance. Diabetes. 2011;60:177-88.

16. Liu NC, Lin WJ, Kim E, Collins LL, Lin HY, Yu IC, et al. Loss of TR4 orphan nuclear receptor reduces phosphoenolpyruvate carboxykinase-mediated gluconeogenesis. Diabetes. 2007;56:2901-9.

17. Kim E, Yang Z, Liu NC, Chang C. Induction of apolipoprotein E expression by TR4 orphan nuclear receptor via 5' proximal promoter region. Biochem Biophys Res Commun. 2005:328:85-90.

18. Kim E, Xie S, Yeh SD, Lee YF, Collins LL, Hu YC, et al. Disruption of TR4 orphan nuclear receptor reduces the expression of liver apolipoprotein E/C-I/C-II gene cluster. J Biol Chem. 2003;278:46919-26.

19. Li G, Lee YF, Liu S, Cai Y, Xie S, Liu NC, et al. Oxidative stress stimulates testicular orphan receptor 4 through forkhead transcription factor forkhead box O3a. Endocrinology. 2008;149:3490-9.

20. Collins LL, Lee YF, Ting HJ, Lin WJ, Liu NC, Meshul CK, et al. The roles of testicular nuclear receptor 4 (TR4) in male fertility-priapism and sexual behavior defects in TR4 knockout mice. Reprod Biol Endocrinol. 2011;9:138.

21. Mu X, Lee YF, Liu NC, Chen YT, Kim E, Shyr CR, et al. Targeted inactivation of testicular nuclear orphan receptor 4 delays and disrupts late meiotic prophase and subsequent meiotic divisions of spermatogenesis. Mol Cell Biol. 2004;24:5887-99.

22. Xie S, Lee YF, Kim E, Chen LM, Ni J, Fang LY, et al. TR4 nuclear receptor functions as a fatty acid sensor to modulate CD36 expression and foam cell formation. Proc Natl Acad Sci U S A. 2009;106:13353-8.

23. Mahajan S, Dkhar HK, Chandra V, Dave S, Nanduri R, Janmeja AK, et al. Mycobacterium tuberculosis modulates macrophage lipid-sensing nuclear receptors PPARgamma and TR4 for survival. J Immunol. 2012;188:5593-603.

24. Deng X, He G, Levine A, Cao Y, Mullins C. Adenovirus-mediated expression of TIMP-1 and TIMP-2 in bone inhibits osteolytic degradation by human prostate cancer. Int J Cancer. 2008;122:209-18.

25. Wilson MJ, Sellers RG, Wiehr C, Melamud O, Pei D, Peehl DM. Expression of matrix metalloproteinase- 2 and -9 and their inhibitors, tissue inhibitor of metalloproteinase- 1 and -2 , in primary cultures of human prostatic stromal and epithelial cells. J Cell Physiol. 2002;191:208-16.

26. Brehmer B, Biesterfeld S, Jakse G. Expression of matrix metalloproteinases (MMP-2 and -9) and their inhibitors (TIMP-1 and -2) in prostate cancer tissue. Prostate Cancer Prostatic Dis. 2003;6:217-22.

27. Castellano G, Malaponte G, Mazzarino MC, Figini M, Marchese F, Gangemi P, et al. Activation of the osteopontin/matrix metalloproteinase-9 pathway correlates with prostate cancer progression. Clin Cancer Res. 2008;14:7470-80.

28. Oh WK, Vargas R, Jacobus S, Leitzel K, Regan MM, Hamer P, et al. Elevated plasma tissue inhibitor of metalloproteinase-1 levels predict decreased survival in castration-resistant prostate cancer patients. Cancer. 2011;117:517-25.

\section{Submit your next manuscript to BioMed Central and take full advantage of:}

- Convenient online submission

- Thorough peer review

- No space constraints or color figure charges

- Immediate publication on acceptance

- Inclusion in PubMed, CAS, Scopus and Google Scholar

- Research which is freely available for redistribution 